\title{
Género, equidad y paz en Guatemala, 1996-2015
}

Artículo de investigación

https://doi.org/

Historia del artículo:

Recibido: 11/09/2020

Evaluado: 09/01/2021

Aprobado: 20/01/2021

\section{Cómo citar este artículo:}

Rouanet Guzmán de Núñez, Rina Patricia "Género, equidad y paz en Guatemala, 1996-2015" Revista Historia de la Educación Latinoamericana vol.23 no.36 (2021)

\section{Rina Patricia Rouanet Guzmán de Núñez ${ }^{1}$}

Universidad San Carlos de Guatemala, Guatemala https://orcid.org/0000-0001-8949-5756

\section{Resumen}

Objetivo: el estudio expone las iniciativas estatales a favor de las mujeres, derivadas de los Acuerdos de Paz en Guatemala; asimismo, destacar analíticamente algunas de las debilidades identificadas y los aspectos estructurales que influyen de manera adversa en su implementación y resultados.

Originalidad/ aporte: constituye principalmente la revisión comparativa de las políticas públicas, que proporciona referentes para futuras iniciativas o para mejorar las existentes.

Método: la investigación es de naturaleza cualitativa, de carácter descriptivo y analítico.

1 Máster en Administración Educativa de la Universidad del Valle de Guatemala. Doctoranda en Historia y Estudios Humanísticos: Europa, América, Arte y Lenguas en la Universidad Pablo de Olavide, España. Grupo de Estudio de Formación Docente, USAC. rinarouanet3@gmail.com 
Estrategias de interpretación basadas en los enfoques hermenéutico y holístico, por la necesidad de identificar el recorrido de las mujeres y sus conquistas, pero también considerando la multidimensionalidad del problema.

Conclusión: del estudio que aún existen normas jurídicas que legitiman prácticas discriminatorias hacia las mujeres y la institucionalidad de género requiere el fortalecimiento de sus instancias para cumplir con el mandato misional. Las Políticas Públicas han evolucionado favorablemente en cuanto a su diseño y gestión estratégica, pero su efectiva implementación e incidencia aún no queda evidenciada. Las asimetrías de género requieren cambios tanto en las prácticas institucionales como en las relaciones sociales en las que la desigualdad es una normalidad.

Palabras clave: Acuerdos de Paz; mujer; género; igualdad.

\section{Gender, equitiy and peace in Guatemala, 1996-2015}

\section{Abstract}

Objective: the study exposes the state initiatives in favor of women, derived from the Peace Agreements in Guatemala; it also analytically highlights some of the weaknesses identified and the structural aspects that adversely influence their implementation and results.

Originality/support: it is mainly the comparative review of public policies, which provides references for future initiatives or to improve the existing ones.

Method: the research is qualitative in nature, descriptive and analytical.

Strategy/ information gathering:Interpretation strategies based on the hermeneutic and holistic approaches were used, due to the need to identify the path of women and their achievements, but also considering the multidimensionality of the problem.

Conclusions: from the study that there are still legal norms that legitimize discriminatory practices against women and that gender institutions need to be strengthened in order to comply with their mission mandate. Public policies have evolved favorably in terms of their design and strategic management, but their effective implementation and impact is not yet evident. Gender asymmetries require changes both in institutional practices and in social relations in which inequality is a normality.

Keywords: Peace Agreements; women; gender; equality. 


\section{Gênero, igualdade e paz na Guatemala, 1996-2015}

\section{Resumo}

Objectivo: o estudo expõe iniciativas estatais a favor das mulheres, derivadas dos Acordos de Paz na Guatemala; também destaca analiticamente algumas das fraquezas identificadas e os aspectos estruturais que influenciam negativamente a sua implementação e resultados.

Originalidade/aporte: é principalmente a revisão comparativa das políticas públicas, que fornece referências para iniciativas futuras ou para melhorar as existentes.

Método: a investigação é de natureza qualitativa, descritiva e analítica.

Estratégia/coleta de dados: Foram utilizadas estratégias de interpretação baseadas em abordagens hermenêuticas e holísticas, devido à necessidade de identificar o percurso das mulheres e as suas realizações, mas também considerando a multidimensionalidade do problema.

Conclusões: O estudo mostra que ainda existem normas legais que legitimam práticas discriminatórias contra as mulheres e que as instituições de género precisam de ser reforçadas a fim de cumprirem o seu mandato de missão. As políticas públicas evoluíram favoravelmente em termos da sua concepção e gestão estratégica, mas a sua efectiva implementação e impacto ainda não é evidente. As assimetrias de género exigem mudanças tanto nas práticas institucionais como nas relações sociais em que a desigualdade é normal.

Palavras-chave: Acordos de Paz; mulheres; género; igualdade.

\section{Introducción}

La finalización del conflicto armado interno en Guatemala, que duró 36 años y la consecuente firma de los Acuerdos de Paz, constituyó una oportunidad para superar las profundas desigualdades entre sus habitantes, mejorar las condiciones de vida y provocar oportunidades de desarrollo para todas y todos sus habitantes. En el caso de las mujeres, evitar su marginación, discriminación y las inequidades de las que han sido víctimas debido a la cultura patriarcal prevalente en la sociedad guatemalteca.

Derivado de los compromisos adquiridos por el Estado Guatemalteco, se ha implementado una serie de iniciativas encaminadas al respeto y defensa de los derechos de las mujeres, así como a promover su desarrollo. Entre tales iniciativas se destacan: la producción y modificación de leyes, la creación de mecanismos para la institucionalización del género y la formulación de Políticas Públicas destinadas a materializar la igualdad de oportunidades 
y el desarrollo para las mujeres; pese a estos esfuerzos, los indicadores continúan reflejando su desventaja ante los hombres.

El objetivo del presente artículo de reflexión, es exponer dichas iniciativas y destacar analíticamente algunas de las debilidades, no solo porque las iniciativas constituyen una conquista para las mujeres guatemaltecas en el marco de la paz, sino también porque es oportuno reflexionar acerca de los aspectos estructurales que influyen de manera adversa en su implementación. El ejercicio investigativo de naturaleza cualitativa-interpretativa, puede aportar algunos referentes para futuras iniciativas o para mejorar las ya existentes.

Se pudo determinar que existe nutrida información sobre lo que se ha hecho por parte del Gobierno, no obstante, la falta información respecto a evaluaciones de impacto y de los resultados alcanzados con las iniciativas en favor de las mujeres, deja la incertidumbre si se ha avanzado en el camino correcto.

La revisión documental, aportó elementos teóricos en cuanto a las acciones estratégicas para la inclusión de la igualdad de género en la actuación gubernamental, la institucionalización de género y la planificación con enfoque de género. Por otra parte, la revisión de los indicadores y la indagatoria con especialistas, ha develado que, si bien es cierto, hay esfuerzos por parte del Estado para contrarrestar la discriminación, exclusión y el trato inequitativo, además de haberse posicionado la problemática de las mujeres en la agenda pública; la igualdad de género requiere cambios en las prácticas institucionales y en las relaciones sociales en las que subyacen patrones de inequidad y desigualdad.

Es concluyente que aún existen normas jurídicas que legitima prácticas discriminatorias hacia las mujeres; la institucionalidad de género requiere del fortalecimiento de sus instancias para cumplir con el mandato misional; las Políticas Públicas han evolucionado favorablemente en cuanto a su diseño y gestión estratégica, pero su efectiva implementación e incidencia aún no queda evidenciada.

\section{Un poco de contexto}

Tal y como lo han descrito, con cierto toque de poesía, los apuntes característicos del país: si hay algo que define a Guatemala son los contrastes. ${ }^{2}$ Guatemala es la cuna del Premio Nobel de Literatura 1967: Miguel Ángel Asturias, pero actualmente el 18.5 \% de habitantes no sabe leer ni escribir. ${ }^{3}$ Es patria de la Premio Nobel de la Paz 1992, Rigoberta Menchú, pero diariamente ocurren muertes causadas por la violencia homicida, cuya tasa es de 23.5 por cada 100,000 habitantes, ${ }^{4}$ condición que nos sitúa entre los países más violentos de la región.

2 Ana Lucía González, Un país de Contrastes (Guatemala, Revista D, 2015) https://www.prensalibre.com/revista-d/ contrastes_de_guatemala-disparidad-indicadores_economicos_y_sociales_0_1307269420/ (20/3/2020)

3 INE, Censo Nacional de Población 2018. (Guatemala: INE, 2019) https://www.censopoblacion.gt/cuantossomos $(20 / 3 / 2020)$.

4 Programa Diálogos, Informe Estadístico de la Violencia en Guatemala (Guatemala: Diálogos, 2018) https://www. dialogos.org.gt/wp-content/uploads/2018/07/Informe-SEMESTRAL-sobre-la-Violencia-Homicida-en-Guatemala-2018-ver-FINAL.pdf ( 22/04/2020) 
Guatemala fue cuna de la civilización maya que hizo aportes muy significativos a la arquitectura, la matemática, la topografía, el arte textil, etc. que se han proyectado a través de los siglos. Paradójico resulta que hoy en día, las comunidades indígenas guatemaltecas son las más atrasadas y carecen de acceso igualitario a la educación, salud o servicios básicos, respecto del resto de población, mayoritariamente mestiza. Además de marginados, los indígenas fueron víctimas de políticas de exterminio durante la guerra civil guatemalteca de 1960 a 1996. ${ }^{5}$ Como esas, podría seguirse enumerando las contraposiciones del país en muchos aspectos más, pero hablemos mejor de las disparidades, que abandona la poesía inicial para convertirse en crudas realidades.

En su contexto, Guatemala es reconocida por su rica y diversa cultura; está conformada por cuatro grandes pueblos: maya, xinca, garífuna y ladino. ${ }^{6}$ Cuenta con una población de 16,3 millones de habitantes de acuerdo con el último censo de población, ${ }^{7}$ de la que las mujeres corresponden el $51.5 \%$ de toda la población. El 54 \% de la población vive en el área urbana. En cuanto a grupos étnicos, la población indígena, corresponde al 44 \%. Guatemala sigue siendo un país con altos porcentajes de pobreza total, $59.3 \%$. La pobreza está presente mayoritariamente en la población rural, indígena, mujeres y en los menores de 18 años. ${ }^{8}$ Si bien es cierto, en los últimos años la niñez se ha beneficiado de progresos en las esferas de salud y educación, la inversión social, en Guatemala sigue siendo una de las más bajas de América Latina, lo que dificulta que el país desarrolle programas sociales significativos o de impactos visibles para grupos vulnerables y en particular para los niños y las mujeres.

Según lo describe Juan Pablo Ozaeta del Instituto Centroamericano de Estudios Fiscales, "la pobreza en Guatemala tiene un rostro rural e indígena, pero también de mujer, porque son las niñas las más expuestas a su impacto, especialmente en relación con la oportunidad de tener acceso a la educación". ${ }^{9}$

Políticamente, es un país con un poco más de 20 años de recuperación postconflicto, con una democracia relativamente joven, reinaugurada en el año 1985, cuando luego de gobiernos militares, se instituyó un gobierno civil electo libremente en las urnas. ${ }^{10}$ Casi diez años más tarde, en 1996, con la firma de los Acuerdos de Paz, se puso fin a 36 años de conflicto armado interno y se marcó el inicio de una nueva etapa en la historia nacional.

5 Anónimo, "Los Mayas del esplendor a la miseria. Los descendientes de los mayas viven, discriminados, explotados y sumidos en la miseria", (Colombia: El Tiempo, 2012) https://www.eltiempo.com/archivo/documento/CMS$12467341(23 / 06 / 2020)$.

6 Se emplea el término ladino y no mestizo, dado es el término que se ha empleado en los diferentes censos del Instituto Nacional de Estadística en Guatemala, como una categoría de identificación con fines estadísticos.

7 INE, Censo Nacional de Población 2018. (Guatemala: INE, 2019) https://www.censopoblacion.gt/cuantossomos $(20 / 3 / 2020)$.

8 INE, Encuesta Nacional de Condiciones de Vida: Desigualdad: (Guatemala: INE, 2014) https://www.ine.gob.gt/sistema/uploads/2016/02/03/bWC7f6t7aSbEl4wmuExoNROoScpSHKyB.pdf (16/06/2020)

9 Anónimo, "Pobreza se agudizó en Guatemala" (Guatemala: Prensa Libre, 2016) http://www.prensalibre.com/guatemala/comunitario/expertos-afirman-que-pobreza-se-agudizo-en-guatemala-en-ultima-decada (11/07/ 2020)

10 Más de dos millones de guatemaltecos fueron llamados a las urnas para elegir nuevo presidente de la República entre ocho candidatos que se postularon para el periodo 1986-1991. Después de la revolución de 1944 cuando fue electo democráticamente Juan José Arévalo, Guatemala tuvo gobiernos militares dictatoriales. 
Los Acuerdos de Paz contienen compromisos orientados fundamentalmente a establecer el Estado de derecho en Guatemala, a construir y fortalecer la democracia y las posibilidades de una amplia participación ciudadana en los asuntos de la nación; pero sobre todo, a superar las profundas exclusiones y desigualdades sociales, económicas, culturales y políticas que han caracterizado al país y que han afectado de manera más directa a las mujeres, a la población indígena y a quienes habitan en el área rural.

A través de varios de los Acuerdos, se puso de manifiesto la relevancia de la participación ciudadana, mediante el ejercicio de sus derechos políticos, de la función legislativa, la efectiva administración de la justicia y el garantizar la seguridad ciudadana que, en conjunto, son decisivas para el goce de las libertades y los derechos ciudadanos de toda la sociedad. De igual manera, los Acuerdos de Paz abordaron temas centrales como el trabajo, la tierra, acceso a la educación, salud y la necesidad de que la mujer participe ampliamente en la construcción de la democracia mediante el pleno ejercicio de sus derechos civiles y políticos.

Pese a que, en la actual Carta Magna, ${ }^{11}$ se estableció que hombres y mujeres tienen igualdad ante la Ley, ante la aplicación de la justicia y queda explicita la prohibición a la discriminación étnica o de género, la condición de las mujeres, sigue atada a modelos culturales, que reproducen desigualdades, inequidades y jerarquías patriarcales, dando como resultado, una situación sistemática de desventaja para las mujeres.

Los fenómenos de discriminación étnica y exclusión territorial se suman y combinan con la discriminación de género, limitando de manera amplia y estructural el goce de los derechos ciudadanos y el desarrollo de las potencialidades de las mujeres. ${ }^{12}$ Este entorno, justifica plenamente que se enfoquen los máximos esfuerzos para garantizar iguales oportunidades y disminuir las brechas entre géneros, así como entre mujeres de diferente pertenencia étnica.

De acuerdo con el Informe Mundial sobre la desigualdad de género, que mide la equidad en diversos ámbitos de la población, entre ellos en la política, en educación, el empleo y el acceso a la salud, Guatemala sigue siendo el país con menos equidad en la región; ${ }^{13}$ situación que vale la pena profundizar, ya que, pese a las muchas iniciativas por alcanzar la igualdad de género, su avance parece estar muy distante de lo esperado.

En un recorrido histórico, la lucha reivindicativa de las mujeres en Guatemala, ha buscado su inclusión en los distintos ámbitos de participación social, ciudadana y política, situación que se puede ir contrastando con la incorporación explícita de su participación y trato igualitario en la carta magna, Acuerdos de Paz, instrumentos normativos, políticas y leyes específicas, lo que constituye la respuesta formal a esa lucha y el compromiso asumido por el Estado, ante los compromisos nacionales e internacionales que han impactado directamente en la visibilización de los derechos de las mujeres.

11 La actual Carta Magna (Constitución de la República) en Guatemala fue aprobada el 31 de mayo de 1985 y entró en vigencia el 14 de enero de 1986; los constituyentes la dividieron en tres partes: dogmática, orgánica y pragmática.

12 Segeplan; Igualdad de Género y Empoderamiento de las Mujeres en el Marco del Cumplimiento de Los objetivos del Milenio (Guatemala: Segeplan, 2016) http://www.guatemalaun.org/bin/documents/IGUALDAD_DEGC89NERO_ECOSOC2005052010_final20doc20(2).pdf (29/01/20)

13 Anónimo, Las cifras que reflejan la situación de las mujeres en Guatemala, (Guatemala: Soy 502, 2016) http://www. soy502.com/articulo/cifras-reflejan-situacion-mujeres-guatemala-0 (28/01/20) 
La historia guatemalteca nos muestra que, en materia de participación política y ciudadana, elemento determinante en un sistema democrático, las mujeres han evolucionado escasamente, por lo que es preciso no solo la re conceptualización de su rol, que se ve afectado por un patrón patriarcal, sino también los alcances del ejercicio ciudadano. ${ }^{14}$

La conquista del voto constituye el signo de mayor visibilidad en cuanto a su participación en la vida pública del país. El derecho a sufragio fue reconocido a las mujeres en la Constitución de 1945, pero estuvo restringida la participación a mujeres analfabetas. ${ }^{15}$ Asimismo, en el artículo 136 de la constitución de 1985 relativo a cargos de elección popular se establece que las mujeres tienen los mismos derechos que los hombres para optar a este tipo de cargos $^{16}$; no obstante, en las elecciones del 2011, año de referencia por la cercanía a la conclusión de la Agenda del Milenio, apenas se logró el 12.6 \% de las diputaciones en el Congreso de la República, (18 de 158 escaños). El porcentaje de las mujeres indígenas electas disminuyó de 2.53 \% en el 2007 a 1.89 \% en el 2011. En el gobierno municipal, sólo siete alcaldías (2 \%) fueron ocupadas por mujeres de un total de 336, una más en comparación con las elecciones del año 2007, pero ninguna fue ocupada mujeres indígenas. ${ }^{17}$

En materia de garantías constitucionales, se establece que la vida, la libertad, la propiedad y otros derechos considerados básicos en varios artículos de la Constitución Política de la República, gozan de igual protección hombres como mujeres. Constituyen además, compromisos concretos en los Acuerdos de Paz, pero esta igualdad no se visualiza en la práctica, dado que aún existen leyes que discriminan a la mujer y los indicadores demuestran profundas desigualdades entre hombres y mujeres.

La década previa a la firma de los Acuerdos de Paz en Guatemala y previo a la apertura democrática de 1985, se instalaron mecanismos, entre los que cabe destacarse la creación de la Oficina Nacional de la Mujer, desde la que se impulsaron reformas jurídicas a favor de las mujeres y se recogieron sus demandas para considerarlas en los Acuerdos de Paz. La época posterior es marcada por los avances en la institucionalización de género, producción de políticas públicas para la promoción y el desarrollo integral de las mujeres y la emisión de leyes que respalden el respeto por los derechos de las mujeres.

En el marco internacional, en el período de estudio, se dan movimientos de gran impacto cuya influencia para el país es determinante. Los indicadores establecidos en la Declaración del Milenio en la que se reconoce la necesidad de impulsar igualdad de género y el empoderamiento de la mujer para alcanzar los Objetivos del Milenio ${ }^{18}$ y en la Agenda 2030,

14 Gladys Torres Ruiz, "Feminismo y Derechos humanos en la ciudadanía de las mujeres" (México: CIMAC, 2009) https://cimacnoticias.com.mx/noticia/feminismo-y-dh-vitales-en-ciudadania-plena-de-las-mujeres/ (4/6/2020)

15 La Constitución promulgada en marzo de 1945, garantizó la libre organización de partidos (Artículos 32 y 33 ) y reconoció la ciudadanía a las mujeres con sus derechos y deberes inherentes (Artículo 9), entre otras cosas.

16 Camila Alarcón, La Evolución política de las mujeres en nuestro país. (Guatemala: Plaza Pública, 2011) https://www. plazapublica.com.gt/content/la-evolucion-politica-de-la-mujer-en-nuestro-pais-0. (22/01/20).

17 Sofía Vásquez, Patricia Galicia y Silvia, Monzón; Entre la Realidad y El Desafío: Mujeres y la Participación Política en Guatemala (Guatemala: NDI/Onu Mujeres, 2013).

18 Rosa Bravo, "Las Metas del Milenio y la Equidad de Género" (Santiago de Chile: Cepal, 2004) http://repositorio.cepal. org/bitstream/handle/11362/5920/S045326_es.pdf (28/03/2020) 
que busca lograr la igualdad entre los géneros y empoderar a todas las mujeres y niñas, ${ }^{19}$ constituyen un referente para determinar el avance.

Pese a las acciones promovidas a favor de las mujeres, la discriminación y la disparidad entre los géneros, sigue dándose, impidiendo el pleno goce de los derechos de las mujeres guatemaltecas, además de posicionar al país como uno de los países latinoamericanos con mayor disparidad de género. ${ }^{20}$ Aún existen grandes brechas de oportunidad y el impacto de lo actuado no se evidencia de manera significativa. Aún existe déficit de acceso en los servicios básicos para las mujeres y las niñas. La violencia sexual, intrafamiliar y laboral contra la mujer sigue dándose de manera alarmante. Existe una débil institucionalización de las políticas de género, quedando muchas de las normas y compromisos reducidos a papel y a letra muerta.

\section{Algunas iniciativas estatales derivadas de la firma de la paz en Guatemala}

Los Acuerdos de Paz, como se ha señalado, constituyeron una invaluable oportunidad para Guatemala, ya que juzgaron y pusieron en evidencia los elementos estructurales que históricamente han generado pobreza, desigualdad e inequidad económica, racismo y discriminación cultural, violencia, intolerancia y persecución política. Los mismos trazaron una ruta de reformas y cambios democráticos, inspirados en el anhelo de un país diferente; de una nueva Guatemala. Este fue el primer escaño, para visibilizar, reconocer pública y políticamente la aportación de las mujeres en los diversos procesos socio económico y políticos del país.

Con la firma de los Acuerdos de Paz Firme y Duradera en diciembre de 1996, inicia otra etapa, para la consolidación de la democracia y una esperanza para los grupos más vulnerables. Sin duda alguna, los Acuerdos constituyeron una idónea plataforma para seguir avanzado en el cumplimiento de los compromisos adquiridos a nivel internacional en materia de igualdad de género y respeto a los derechos de las mujeres.

En el contenido de los Acuerdos de Paz, quedó visibilizada la necesidad de propiciar la participación e inclusión de las mujeres indígenas y no indígenas, como agentes importantes en el desarrollo económico-social. En virtud de lo cual se adquieren compromisos para superar las inequidades existentes y garantizar una nueva ciudadanía, con una cultura basada en la equidad y en la corresponsabilidad entre hombres y mujeres, respetando la diversidad como pertenencia étnica, clase social y edad. ${ }^{21}$ De las iniciativas derivadas de los compromisos adquiridos por el Estado guatemalteco se resaltan las siguientes:

19 ONU, Objetivos de Desarrollo sostenible No. 5. (Nueva York: ONU, 2012) http://www.un.org/sustainabledevelopment/es/gender- equality/ (14/01/2020)

20 CEPAL, "Índice global de Brecha de Género Guatemala” ( Santiago de Chile: Cepal, 2018) https://datosmacro.expansion.com/demografia/indice-brecha-genero-global/guatemala (30/03/2020)

21 SEGEPLAN," Informe de Estado de Cumplimiento de la Declaración y Plataforma de Beijing”, (Guatemala: Segeplan, 2014) 


\section{Reformas jurídicas y surgimiento de nuevas leyes}

El Estado incorpora en la normativa jurídica e institucional medidas que responden a los compromisos asumidos frente al pueblo de Guatemala, que impulsan el desarrollo de los derechos de las mujeres, y que constituyen el referente para las acciones, estratégicas, políticas y operativas que de las mismas se deriven. Algunas de estas acciones incluyen las decisiones políticas que dan vida a la institucionalidad creada por el Estado para el adelanto de la mujer en Guatemala y que fortalecen su posicionamiento. Otras son de índole específica y que se relacionan con la protección de las mujeres.

En la segunda mitad de los años 90 se destacaron diversos esfuerzos de las mujeres organizadas para hacer avanzar en el Congreso de la República, marcos normativos sobre violencia contra las mujeres, derechos sexuales y reproductivos, participación política y la eliminación del racismo y la discriminación, de tal esfuerzo se concretan la Ley contra la violencia intrafamiliar, la Ley de Dignificación y Promoción Integral de la Mujer, ${ }^{22}$ así como algunas reformas al Código Civil: edad para contraer matrimonio, cambio en la representación conyugal ${ }^{23}$ estas reformas al código civil fueron concretadas recientemente.

Asimismo, se plantearon iniciativas de ley en temas como la discriminación étnica, prohibición del servicio militar obligatorio y contra el acoso sexual con agravante si el mismo es para mujeres indígenas. Algunas de tales iniciativas no han sido aprobadas por un Congreso eminentemente masculinizado a decir por su conformación 89\% de hombres.

A partir del año 2001 se reportan otros avances ya que el Congreso de la República aprobó la Ley de Desarrollo Social (Decreto Legislativo 42-2001) y en 2002 la ratificación del Protocolo Facultativo de la Convención para la Eliminación de todas las Formas de Discriminación contra la Mujer (Decreto Legislativo 11-2002). Ese mismo año se avanzó en afianzar la democracia participativa, cuando el Congreso de la República aprobó tres nuevas leyes concebidas como instrumentos para fortalecer la participación ciudadana: Ley General de Descentralización (Decreto 14-2002), Ley de los Consejos de Desarrollo Urbano y Rural (Decreto 11-2002) y Código Municipal (Decreto 12-2002). Esta trilogía de leyes abrió un nuevo ciclo de participación ciudadana para las mujeres, puesto que permitió la organización y participación en las decisiones del desarrollo en los niveles municipal, departamental, regional y nacional. ${ }^{24}$ En este mismo año, se aprobó en el Congreso de la República el Plan de Acción para la plena participación de las mujeres Guatemaltecas 2002-2012, diseñado por el Foro Nacional de la Mujer y la Secretaría Presidencial de la Mujer -Seprem-.

Un año más tarde (2003) el Congreso de la República aprobó la Ley de Idiomas Nacionales, que abrió la posibilidad a las mujeres rurales monolingües de acceder a la justicia

22 Organismo Judicial, "Ley de Dignificación, Promoción Integral de la Mujer (Guatemala: José Pineda Ibarra, 1990)

23 Actualmente, es igual para ambos cónyuges. El Código Civil, fue modificado dado que originalmente la representación conyugal correspondía única y exclusivamente al hombre, pero fue impugnado y posteriormente modificado.

24 Consejos de Desarrollo, en el marco de la democracia representativa, es una herramienta jurídica y política que posibilita la identificación de necesidades para la planificación, para hacer una gestión más aterrizada a la realidad local. 
en sus propios idiomas (Decreto 19-2003). ${ }^{25}$ En el 2005 se aprobó la Ley Marco para el Cumplimiento de los Acuerdos de Paz (Decreto 52-2005) que contempla representación de las mujeres en el Consejo Nacional de los Acuerdos de Paz -CNAP-. En el año 2008, se aprobó la Política y Plan de Equidad de Género en la Educación Superior 2006-2014, en el Instituto Universitario de la Mujer de la Universidad de San Carlos de Guatemala-Iumusacpara incorporar en la vida académica el enfoque de género. ${ }^{26}$

Los años 2008 y 2009 fueron cruciales para las mujeres guatemaltecas, pues el Congreso de la República aprobó la Ley contra el Femicidio y otras Formas de Violencia contra la Mujer (Decreto 22-2008)27 y la Ley contra la Violencia Sexual, Explotación y Trata de Personas (Decreto 9-2009). Un año más tarde, aprobó reformas al Código Municipal (Decreto 12 2002, artículo 95) para la creación de las Oficinas Municipales de la Mujer, que serían las responsables de la atención a las necesidades específicas de las mujeres del municipio y del fomento de su liderazgo comunitario, participación económica social y política. También se estableció que el Concejo Municipal debía velar porque se le asignen fondos para su funcionamiento y el cumplimiento de sus objetivos. ${ }^{28}$

Posteriormente, en el Decreto 22-2010, se añadió el artículo 96 para dotar de presupuesto a las Oficinas municipales de la mujer-OMM-, como un mecanismo para promover a lo interno de las Municipalidades, los derechos de las mujeres, procurando su desarrollo en los diversos espacios. ${ }^{29}$

Del período 2010 a 2017, se registran acciones importantes encaminadas a erradicar la violencia y discriminación contra la mujer con la aprobación y reformas de algunas leyes, tal es el caso de: las reformas al Código Municipal nuevamente, que amplió las funciones de la OMM y elevó de Oficina a Dirección Municipal de la Mujer (Decreto 39-2016), la aprobación de la Ley de búsqueda inmediata de mujeres desaparecidas (Decreto 9-2016), las reformas al Código Civil (Decreto13-2017) relativas a eliminación de las uniones de hecho para menores de edad y elevar la edad del matrimonio a 18 años respectivamente; más recientemente se aprobó la Ley para crear el registro de agresores sexuales (Decreto 22-2017).

No obstante que la aprobación de dichas leyes representan una victoria simbólica para los defensores y las defensoras de los derechos de las mujeres, los expertos han argumentado que es poco probable ver un cambio significativo en la situación de las mujeres porque las leyes fueron aprobadas sin alterar la forma en que las mujeres están vistas por la sociedad,

25 Academia de Lenguas Mayas, "Ley de Idiomas Nacionales" (Guatemala: ALMG, 2003) https://www.acnur.org/fileadmin/Documentos/BDL/2008/6731.pdf (6/06/2020)

26 Con la finalidad de que se reconozca, promueva y garantice la igualdad de oportunidades para las mujeres y hombres en el acceso a una educación superior.

27 Ley Contra el Femicidio y otras formas de violencia contra la mujer, que específicamente tipifica la muerte de una mujer por razones de género basada en las relaciones desiguales de poder y ha reformado la estructura administrativa del sector justicia, salud y educación.

28 Congreso de la República, "Decreto Ley 12-012, Código Municipal” (Guatemala: CR, 2012) https://www.acnur.org/ fileadmin/Documentos/BDL/2008/6698.pdf (11/07/2020)

29 Congreso de la República; Decreto Ley 022-2010, "Creación de las Oficinas Nacionales de la Mujer" (Guatemala: CR, 2010). www.congreso.gob.gt (11/07/2020) 
la desigualdad normalizada entre los hombres y las mujeres y la autoridad de los hombres que se mantiene en las prácticas sociales y en algunas de las leyes. ${ }^{30}$

Una de las leyes con más impacto para las mujeres ha sido la Ley de Femicidio por la forma en que se ha operativizado su administración; se puede afirmar que en la actualidad hay persecución penal para los infractores; se ha creado la infraestructura institucional para hacer operativa la norma y los protocolos de actuación para atención de las víctimas. En contraposición a ello, falta propiciar una mayor cultura de denuncia, ya que muchas mujeres experimentan miedo o vergüenza en acudir ante las instancias correspondientes a exponer sus casos. Más aún en el área rural y en poblaciones indígenas.

Es plausible la producción legislativa desde la firma de la paz hasta el momento actual, pero es preciso resaltar que Guatemala aun cuenta con varias leyes discriminatorias hacia las mujeres. El problema se agudiza, cuando incluso las mismas leyes diseñadas para proteger a las mujeres, siguen siendo interpretadas por gentes del Estado que perpetúan estereotipos de género, situación que a menudo ha perjudicado principalmente a las adolescentes y jóvenes. El ejemplo más claro, se encuentra en el Código Civil de Guatemala ${ }^{31}$ que contiene diferentes artículos en los que se encuentran estereotipos y una evidente discriminación. Algunos ya han sido modificados, pero para el análisis ha sido importante identificarlos. Seguidamente se detallan algunos de los extremos afirmados:

Artículo 81. "Este regula que la mayoría de edad determina la libre aptitud para contraer matrimonio, sin embargo, pueden contraerlo el varón mayor de dieciséis años y la mujer mayor de catorce"

Esta norma fue derogada recientemente, modificándose la edad de contraer matrimonio hasta los 18 años, tanto hombres como mujeres. A pesar de la obviedad en cuanto a las razones para la modificación de la norma, la estadística aporta cifras alarmantes en cuanto a embarazos en adolescentes lo que pone en riesgo su salud e interrumpe su desarrollo emocional.

Artículo 89. "No puede ser autorizado el matrimonio de la mujer antes de que transcurran trescientos días contados desde la disolución del anterior matrimonio, o de la unión de hecho o desde que se declare nulo el matrimonio..."

La prohibición refiere únicamente a la mujer, lo que constituye una explícita discriminación; independientemente que el espíritu de esta prohibición, es el evitar una confusión de paternidad en caso la mujer pudiera estar embarazada; el argumento se debilita si se consideran los avances de la ciencia en cuanto a poder determinar mediante la prueba de ADN la relación filial. Es concluyente que el Artículo en mención constituía una violación al principio de igualdad, plasmado en la Constitución Política de la República de Guatemala.

Artículo 113. "La mujer podrá desempeñar un empleo, ejercer una profesión, industria, oficio o comercio cuando ello no perjudique el interés y cuidado de los hijos ni las demás atenciones del hogar".

30 Naciones Unidas, "Situación de la mujer en Guatemala” (Guatemala: Redlac, 2017) https://tbinternet.ohchr.org/Treaties/CEDAW/Shared\%20Documents/GTM/INT_CEDAW_NGO_GTM_29095_S.pdf (22/06/2020)

31 Organismo Judicial, Código Civil, Decreto Ley 8-2015, (Guatemala: Congreso de la República, 2015) 
Refleja claramente el arraigo patriarcal imperante en nuestra cultura, atribuyendo con especificidad los roles domésticos a la mujer. Este artículo fue enmendado ante la solicitud del colectivo de mujeres y defensores de los derechos humanos.

\section{El Artículo 114. "Faculta al marido para oponerse a que la mujer se dedique a actividades fuera del hogar..."}

Al igual que el artículo anterior evidencia discriminación y también fue derogado.

\section{Artículo 131. "El marido es el administrador del patrimonio conyugal...",}

Es explícita la discriminación contra la mujer, al excluirla de la administración del patrimonio conyugal lo que contraviene y viola el principio de igualdad.

Actualmente la norma dice: "Bajo el régimen de comunidad absoluta o en el de comunidad de gananciales, ambos cónyuges administrarán el patrimonio conyugal, ya sea en forma conjunta o separadamente".

Como estos ejemplos pueden seguirse enumerando otros, que aparecían explícitamente en normativas de alta jerarquía, que a todas luces eran violatorios y contradecían avances importantes en materia de derechos de las mujeres.

Otro aspecto que cabe mencionarse es que hay temas que no se han legislado, tal es el caso del acoso sexual, que no se ha tipificado como delito y aunque existe la Ley de Femicidio que hace alusión a todo tipo de violencias, no es específica acerca del acoso sexual en espacios de trabajo, centros educativos o la calle. No se ha abordado la ley de cuotas para la participación paritaria en el plano político o el ejercicio de cargos públicos electivos, en la más reciente enmienda de la Ley Electoral y de partidos políticos no se refleja dicho extremo. No existe una ley relacionada con el trabajo no remunerado de las mujeres, por lo que ese aporte está invisibilizado.

Si bien es cierto la producción legislativa de los últimos años ha sido significativa para la protección y avance de las mujeres, también es importante señalar que Guatemala necesita la debida aplicación y cumplimiento de los preceptos constitucionales de igualdad y libertad.

\section{Instituciones e instancias (mecanismos para la institucionalización de género)}

La institucionalidad de género está compuesta por un entramado de compromisos internacionales, leyes, mecanismos y actores que han hecho posible la visibilización del problema de las desigualdades de género en la sociedad y el Estado. Esto abarca ámbitos que van desde la construcción de nuevas concepciones sobre las relaciones de género, pasando por la incorporación de las desigualdades entre hombres y mujeres a las agendas públicas, hasta la institucionalización de esta problemática en el Estado. ${ }^{32}$

32 Virginia, Guzmán. La institucionalidad de género en el estado: nuevas perspectivas de análisis, (Santiago de Chile: Cepal, 2001) 
La firma de los Acuerdos de Paz, en 1996, hizo más viable el surgimiento de nuevos mecanismos para la promoción y defensa de los derechos de las mujeres, así como la incorporación de la perspectiva de género al marco legislativo de la paz en temas como violencia intrafamiliar, sexual, trata y explotación, femicidio y otras formas de violencia. Por otra parte, se inicia la discusión del presupuesto público con enfoque de género ${ }^{33}$ y se hace evidente la necesidad de la participación de las mujeres para la consolidación de una verdadera democracia. Para ello se crearon instituciones e instancias específicas responsables de operativizar la legislación creada, así como los compromisos adquiridos para que permitan cambios e impactos positivos en la vida de mujeres, niñas y adolescentes.

Se puede afirmar que se ha fortalecido la institucionalidad en favor de las mujeres con la creación de unidades de género y secretarías de la mujer, tanto en el Organismo Ejecutivo como en el Judicial; asimismo el Organismo Legislativo representado por el Congreso de la República, cuenta dentro de sus comisiones con la de la Mujer y la Familia. Por otra parte, se ha elevado la categoría de las Oficinas Municipales de la Mujer a Direcciones Municipales de la Mujer. ${ }^{34}$

La creación de la Secretaría Presidencial de la Mujer -Seprem- en el año 2000 según Acuerdo Gubernativo 200-2000, y que constituye el mecanismo de la mujer al más alto nivel en el país, es la encargada de asesorar a la Presidencia de la República y a la institucionalidad pública en materia de derechos humanos de las mujeres. Esto no significa que la dependencia goce de autonomía y que goce de jerarquía sobre otros Ministerios y o Secretarías del Organismo Ejecutivo. Según el mandato que le fue conferido, la Seprem es una medida afirmativa para el avance de los derechos de las mujeres, la cual incide en la consolidación del cumplimiento a los compromisos contenidos en los Acuerdos de Paz y los compromisos internacionales ratificados por el Estado de Guatemala. Hasta el momento la Seprem, es el ente rector en políticas públicas para el desarrollo integral de las mujeres.

Aunque es positiva su existencia, como primer paso en los mecanismos institucionales para coordinar instancias del Estado relacionadas con la situación y condición de la mujer, hay expertos que sugieren que ésta deberá constituirse en una instancia de transición hacia la creación del Instituto rector de las Políticas Públicas para la Mujer, afirma el informe sombra de derechos de las mujeres. ${ }^{35}$

Se han creado instancias de coordinación interinstitucional como la Coordinadora nacional para la prevención de la violencia intrafamiliar y contra las mujeres -Conaprevi-, que fue instaurada también en el año 2000, tomando como referente los compromisos asumidos por el Estado de Guatemala a nivel internacional en materia de erradicación de la violencia contra la mujer y en el marco de la responsabilidad por asegurar una vida libre de violencia.

33 Los presupuestos públicos no son neutrales al género. Todo gasto tiene algún impacto, el cual se evidencia de manera diferenciada en mujeres y en hombres. No tomar en cuenta este aspecto, no significa neutralidad, sino desconocimiento a las diferencias de género y supone una inadecuada distribución de los recursos. Holfauer, citado en, Rina Rouanet y Rebeca González, "Presupuesto y Proyectos con enfoque de género" (Guatemala: Serviprensa, 2003).

34 Decreto Ley 39-2016 creación de las Direcciones Municipales de la Mujer.

35 Nizkor. "Informe Sombra Sobre la Situación de los Derechos Humanos de las Mujeres en Guatemala" (Guatemala: Nizkor, 2001) http://www.derechos.org/nizkor/guatemala/doc/sombra.html (30/05/2020) 
La Coordinadora nacional para la prevención de la violencia intrafamiliar y contra la mujer -Conaprevi- se constituyó como una instancia con carácter coordinador, asesor e impulsor de las políticas públicas relativas a reducir la violencia intrafamiliar y la violencia en contra de las mujeres. La misma se conformó por representantes del sector público y de sociedad civil. No obstante, a partir de 2012 se inicia un proceso de debilitamiento de la Coordinadora hasta lograr su inoperancia durante el periodo 2013-2015, lo cual conllevó a organizaciones de sociedad civil a presentar una denuncia en contra del Estado de Guatemala por violación de Derechos ante la Corte Interamericana de Derechos Humanos -CIDH-.

Otra instancia derivada de la firma de los Acuerdos de Paz y que constituye un rasgo visible de la institucionalidad del tema, fue la creación de la Defensoría de la mujer indígena DEMI, cuya creación obedece a un compromiso realizado en el Acuerdo sobre identidad y derechos de los pueblos indígenas por el gobierno de Guatemala, en donde se comprometió a promover la divulgación y fiel cumplimiento de la Convención sobre la sobre la eliminación de todas las formas de discriminación contra la mujer y de otros instrumentos Internacionales relativos a los derechos de los pueblos indígenas, ratificados por Guatemala.

La Defensoría de la mujer indígena DEMI fue creada mediante Acuerdo Gubernativo número 525-99 de fecha 19 de julio de 1999, como dependencia de la Presidencia de la República, con capacidad de gestión y ejecución administrativa, técnica y financiera, con el fin de atender las particulares situaciones de vulnerabilidad, indefensión y discriminación de la mujer indígena, para lo cual deberá promover las acciones de la defensa y pleno ejercicio de sus derechos; adscrita inicialmente a la Comisión Presidencial Coordinadora de la Política del Ejecutivo en materia de Derechos Humanos -Copredeh- que posteriormente como logro adquiere su autonomía con el Acuerdo gubernativo 442-2007 del 25 de septiembre de $2007 .{ }^{36}$ Más adelante en el año 2010 el gobierno de turno pretendió fusionarla con la Secretaría de pueblos indígenas del Organismo Judicial, (anteriormente Unidad de pueblos indígenas) considerando afinidad o duplicidad de funciones, pero desde los grupos organizados y defensores de las mujeres indígenas se estaría retrocediendo con ello la relativa autonomía lograda, además porque su condición de mujeres indígenas requiere de especial atención en todos aquellos aspectos culturales como el idioma, la identidad, la cosmovisión, que son elementos fundamentales a considerar dentro del procesos de atención a casos, incluyendo los paradigmáticos. ${ }^{37}$

Muchas de estas instituciones se ven gravemente restringidas por falta de financiamiento con relación al extenso mandato que estas asumen. Existe, además, cierta desarticulación institucional, tanto de forma interna como en su labor interinstitucional con Ministerios y otras instancias del gobierno. Adicionalmente, han tenido una capacidad limitada para aplicar y supervisar la ejecución de las políticas de género existentes, con el perjuicio añadido de su escasa presencia a nivel departamental y municipal.

Algunas acciones de los gobiernos de turno, lejos de incidir en la articulación y fortalecimiento de las instituciones existentes, encargadas de velar por los derechos de las mujeres, han provocado duplicidad de esfuerzos y debilitado a las mismas. Por otra parte, la jerarquía

36 DEMI. "Historia y antecedentes" (Guatemala: Demi, 2007) https://www.demi.gob.gt/?page_id=6560 (18/08/2020)

37 Albedrío, "Pronunciamiento político contra la fusión de la Demi" (Guatemala: Albedrío, 2010) http://www.albedrio. org/htm/otrosdocs/comunicados/apom-001.htm (18/08/2020) 
horizontal con la que fueron creadas las instituciones, no les permite ejercer presión para el impulso de las acciones planificadas, sobre otras de igual jerarquía.

En el caso concreto de la Secretaría Presidencial de la Mujer, que es la instancia de más alta jerarquía, denota en su histórico que no todos los gobiernos le han dado un lugar preponderante, incluso ha sido debilitada en su actuación por la falta de presupuesto y apoyo. La Defensoría de la Mujer Indígena tiene un amplio mandato, pero su presencia a nivel nacional resulta escasa, igualmente por la falta de presupuesto.

\section{Políticas Públicas para el desarrollo de las mujeres}

Las políticas públicas han sido consideradas, desde las distintas vertientes de la ciencia política, como el resultado del conjunto de procesos mediante los cuales las demandas sociales se transforman en opciones políticas y en toma de decisiones de quienes ejercen la autoridad política. En otras palabras, en decisiones de gobierno.

En materia de políticas públicas específicas para la promoción de los derechos y el avance de las mujeres, generadas en el marco de la paz y dentro de compromisos adquiridos a nivel internacional, las más significativas son: Política nacional y Plan de equidad de oportunidades (1996-2000), Política Nacional de Promoción y Desarrollo de las mujeres guatemaltecas y Plan de equidad de oportunidades (1998-2001), Política Nacional de promoción y desarrollo de las mujeres guatemaltecas (PNPDMG) y Plan de equidad de oportunidades (2001-2006) y las más relevante por su temporalidad es la Política Nacional de Promoción y Desarrollo Integral de las Mujeres PNPDIM (2008-2023), esta versión fue mejorada en 2009 y se le incorporó el Plan de Equidad de Oportunidades, con vigencia hasta el año 2023. La misma se enmarcó en la Ley de Dignificación y Promoción Integral de la Mujer (Decreto Ley Número 7-99 del Congreso de la República y consideró las políticas anteriores e incluso la Política Nacional para el Desarrollo y promoción de la mujer y la familia en Guatemala (1986-1990), que aunque se formuló previo a la firma de los Acuerdos de Paz, contenía ejes de gran trascendencia para las mujeres.

La política vigente, PNPDIM y PEO 2008-2023 constituye la directriz, para la institucionalización de la perspectiva de género y étnica dentro del aparato gubernamental. La misma incorpora en sus 12 ejes, las esferas de preocupación de la Plataforma para la Acción Mundial, los Objetivos de Desarrollo del Milenio; la Declaración de Pueblos Indígenas y los compromisos contenidos en los Acuerdos de Paz.

Está diseñada para articular la misma con los planes institucionales y de gobierno ya que cada uno de los ejes responde a ámbitos relacionados con los problemas con los que enfrenta la mujer: salud, educación, empleo, por mencionar algunos. Esta Política es producto del compromiso por parte del Gobierno de Guatemala en la búsqueda del desarrollo de las mujeres y es producto de los consensos entre Estado y las redes de organizaciones de mujeres y feministas. Asimismo, acumula la experiencia de políticas anteriores que se formularon antes y después de la firma de los Acuerdos de Paz y que han recogido las demandas de muchas mujeres guatemaltecas. 
De acuerdo con algunos indicios y monitoreos realizados por organismos internaciones y la sociedad civil organizada, no todas las dependencias del Organismo Ejecutivo han asumido en la justa dimensión la incorporación de los ejes de la política en su propia planificación, lo que repercute en poca o nula asignación presupuestaria para el tema específico del adelanto de las mujeres.

Las políticas públicas en la tradicional lógica del Estado guatemalteco, han correspondido a una visión de corto plazo, fue hasta la década de los 90 cuando se empieza la formulación de políticas públicas de más largo plazo y son precisamente aquellas que están destinadas a atender a las poblaciones en situación de riesgo y vulnerabilidad.

Aunque se reconoce que el país ha ido avanzado en su capacidad de diseño y formulación de políticas, en especial a partir de la firma de los Acuerdos de Paz, el avance en ciertas capacidades instaladas en cuanto a planificación, se ve opacado con la poca capacidad para la implementación, ejecución y cumplimiento de las metas por parte de las diferentes dependencias involucradas, repercutiendo adversamente en la atención de necesidades de la población

La falta de indicadores en la a mayoría de las políticas para el avance de las mujeres, es un hallazgo que repercute negativamente no solo en su evaluación y acompañamiento sino en las nuevas políticas que se formulen, pues no existe una retroalimentación respecto a los logros, que permita incorporar prácticas de éxito y eficiencia en la gestión de la política. Por otra parte, la discontinuidad de las políticas y programas es la reincidencia de cada cuatro años, tiempo que dura un periodo presidencial.

La política 2008-2023 es la excepción, dado que fue formulada a largo plazo y cuenta con indicadores para determinar los resultados y una medición de impacto. destacándose que goza de legitimidad por el alto grado de consulta y de participación de mujeres en su elaboración, el plazo otorgado para su vigencia y la vinculación del presupuesto con enfoque de género.

\section{Conclusiones}

A través del presente artículo se ha podido describir algunos de los esfuerzos realizados por el Estado Guatemalteco, para el avance y protección de los derechos de las mujeres, en cumplimiento a los compromisos adquiridos con la firma de la paz: emisión de nuevas leyes, formulación de políticas públicas e institucionalización género. Esfuerzos que, de acuerdo con el análisis realizado, no eximen al Estado de las debilidades implícitas en su implementación, ni de los indicadores que posicionan a la mujer en desventaja, aún después de más de dos décadas de concluida la guerra interna que se vivió en Guatemala

Las iniciativas implementadas por el Estado guatemalteco constituyen la formalización de los compromisos adquiridos y son susceptibles de inventariarse, pero no se ha evaluado el impacto o la efectividad de tales iniciativas, por lo que la interpretación de su incidencia ante la problemática que viven las mujeres, no pasará de ser conjetural para los responsables de la gestión pública en este país y para la sociedad en su conjunto. 
En materia legal, los avances para normar la defensa y derechos de las mujeres han sido significativos, no obstante y aunque se han promovido reformas, aún existen leyes en el país que contradicen la igualdad que se persigue, pues mantienen distinciones injustificadas basadas en el género. Por otra parte, existen leyes pendientes de emitirse y que han frenado el avance de ciertos indicadores, por ejemplo: no se ha tipificado el acoso sexual como delito; no existe la ley de cuotas para la participación paritaria en el plano político o el ejercicio de cargos públicos electivos; no existe una ley relacionada con el trabajo no remunerado de las mujeres, por lo que ese aporte está invisibilizado. Es fundamental la debida aplicación y cumplimiento de los preceptos constitucionales de igualdad y libertad.

En lo que respecta a los mecanismos para la igualdad de género, las instancias específicas se ven gravemente restringidas por falta de financiamiento con relación al extenso mandato que estas asumen. Además, existe cierta desarticulación interinstitucional con otras instancias del gobierno, lo cual provoca duplicidad de esfuerzos y distanciamiento de las metas. Adicionalmente, han tenido una capacidad limitada para aplicar y supervisar la ejecución de las políticas de género existentes, no solo por su escasa presencia a nivel departamental y municipal, sino también porque la jerarquía horizontal con la que fueron creadas las instituciones, no les permite ejercer presión para el impulso de las acciones planificadas, sobre otras instituciones de igual jerarquía.

En materia de políticas públicas se puede afirmar que a lo largo de tres décadas las políticas formuladas han evolucionado favorablemente en cuanto a su elaboración técnica, así como también su gestión política y estratégica. Vale aludir la Política Nacional de Promoción y Desarrollo de las Mujeres Guatemaltecas y el Plan de Igualdad de Oportunidades para 20082023, legitimada a través de consultas con la sociedad civil. Constituye uno de los adelantos más significativos en materia de planificación a favor de las mujeres, pues la vigencia que le fue asignada ha permitido que trascienda a través de varios períodos de gobierno, ya que por lo general se han formulado políticas de gobierno más no de Estado.

El ejercicio investigativo condujo a identificar algunos elementos adversos atribuidos a las iniciativas de intervención estatal para mitigar la problemática de las mujeres, lo que pone al descubierto la debilidad de la institucionalidad pública y falta de voluntad política. Se siguen observando brechas y discrepancias que hacen repensar en las políticas impulsadas, revisar los marcos legales, verificar los desaciertos y refundar las instituciones encargadas de velar por los derechos de las mujeres. Pero poco se puede avanzar en tanto prevalezca el arraigo cultural de orden patriarcal predominante en nuestra sociedad, que privilegia a los hombres y discrimina a las mujeres. Esta condición que provoca y admite las desigualdades como algo natural y hasta correcto, ha frenado muchos impulsos que permitan igualdad de oportunidades a las mujeres. Solo para cuando superemos las disparidades de género, de etnia, ideológicas y de cualquier índole podremos decir que se logró la paz en Guatemala. 


\section{Referencias}

Aldana Mendoza, Carlos. “Desafíos pedagógicos de la paz”, Guatemala: Editorial Universitaria, 1995.

Asamblea Nacional Constituyente, Constitución Política de la República de Guatemala, Guatemala: Tipografía Nacional, 1985.

Academia de Lenguas Mayas. Ley de Idiomas Nacionales. Guatemala, ALMG, 2003. https://www.acnur. org/fileadmin/Documentos/BDL/2008/6731.pdf (6/06/2020)

Alarcón, Camila. La Evolución política de las mujeres en nuestro país. Guatemala, Plaza Pública, 2011 https://www.plazapublica.com.gt/content/la-evolucion-politica-de-la-mujer-en-nuestro-pais-0. $(22 / 01 / 20)$.

Albedrío. Pronunciamiento político contra la fusión de la Demi- Guatemala, Albedrío, 2010. http://www. albedrio.org/htm/otrosdocs/comunicados/apom-001.htm (18/08/2020)

Anónimo. Pobreza se agudizó en Guatemala. Guatemala, Prensa Libre, 1916. http://www.prensalibre. com/guatemala/comunitario/expertos-afirman-que-pobreza-se-agudizo-en-guatemala-en-ultima-decada (11/07/ 2020)

Anónimo. "Los Mayas del esplendor a la miseria. Los descendientes de los mayas viven, discriminados, explotados y sumidos en la miseria" Colombia, El Tiempo, 2012. https://www.eltiempo.com/ archivo/documento/CMS-12467341 (23/06/2020).

Barrios-Kleé, W. y Romero, W. Mirando el presupuesto público con perspectiva de género: educación, salud y vivienda. Guatemala, Flacso, 2010.

Bravo, Rosa. Las Metas del Milenio y la Equidad de Género. Santiago de Chile, Cepal, 2004. http://repositorio.cepal.org/bitstream/handle/11362/5920/S045326_es.pdf (28/03/2020)

CEPAL. İndice global de Brecha de Género. Guatemala, Cepal, 2018. https://datosmacro.expansion.com/ demografia/indice-brecha-genero-global/guatemala (30/03/2020)

Comisión de Esclarecimiento Histórico (CEH). "Causas y orígenes del enfrentamiento armado interno. Guatemala, memoria del silencio". Guatemala, Servigráficos, 1999.

CODEFEM. Monitoreo del Gasto Público. Guatemala, Codefem, 2012. http://codefem.org/wp/wp-content/ uploads/2017/06/Monitoreo-Gasto-de Género_2012_CODEFEM.pdf (11/07/2020)

Congreso de la República. Decreto Ley 12-012, Código Municipal. Guatemala, CR, 2012. https://www.acnur. org/fileadmin/Documentos/BDL/2008/6698.pdf (11/07/2020)

Congreso de la República. Decreto Ley 022-2010, Creación de las Oficinas Nacionales de la Mujer. Guatemala, CR, 2010. www.congreso.gob.gt (11/07/2020)

Congreso de la República. Acuerdos de Paz. Guatemala, CR, 1996. http://old.congreso.gob.gt/Docs/PAZ/ Acuerdo.pdf (11/07/2020)

Congreso de la República. Carta Magna 1986. Guatemala, CR, 1987.

http://www.congreso.gob.gt (11/07/2020)

DEMI. Historia y antecedentes. Guatemala, Demi, 2007. https://www.demi.gob.gt/?page_id=6560 (18/08/2020)

Facio, Alda. Los Derechos Humanos desde una Perspectiva de Género y las Políticas Públicas. Otras Miradas. México, Redalyc, 2003. ISSN: 1317-5904. https://www.redalyc.org/articulo.oa?id=183/18330102 (16 de Abril de 2020).

Gomáriz Enrique, García Ana Isabel. Mujeres Centroamericanas: ante la crisis, la guerra y el proceso de paz, Costa Rica, Flacso, 1989. 
González, Ana Lucía- Un país de Contrastes. Guatemala, Revista D, 2015. https://www.prensalibre.com/revista-d/contrastes_de_guatemala-disparidad-indicadores_economicos_y_sociales_0_1307269420/ $(20 / 3 / 2020)$

Guzmán, Virgina. Institucionalización del Género en el Estado: Nuevas Perspectivas de Análisis, Unidad de Mujer y Equidad de Género. Chile, Cepal, 2001.

Instituto Nacional de Estadística. Encuesta Nacional Economía e Ingresos. Guatemala, INE, 2012. https:// www.cepal.org/es/publicaciones/7156-guatemala-poblacion-desarrollo-un-diagnostico-sociodemografico. (11/07/2020)

Instituto Nacional de Estadística. Cuántos Somos, Censo Nacional de Población, 2018. Guatemala, INE, 2018. https://www.censopoblacion.gt/cuantossomos (15/06/2020)

Ministerio de Educación. Segundo Censo Nacional de Talla de Escolares de Primer Grado Primaria. Guatemala, Diplan, 2002.

Ministerio de Educación. Estrategia para la implementación de la Política Nacional de Promoción y Desarrollo Integral de las Mujeres (PNPDIM) y el Plan de Equidad de Oportunidades. Guatemala, Digecor, 2016.

Ministerio de Finanzas Públicas. Formulación de Presupuestos por período. Guatemala, Mifin 2018. https://www.minfin.gob.gt/images/downloads/presupuesto_formulacion/2018-22/estrategia. $\operatorname{pdf}(12 / 06 / 2020)$

Naciones Unidas, Situación de la mujer en Guatemala (Guatemala, Redlac, 2017) https://tbinternet. ohchr.org/Treaties/CEDAW/Shared\%20Documents/GTM/INT_CEDAW_NGO_GTM_29095_S.pdf $(22 / 06 / 2020)$

Nizkor. Informe Sombra Sobre la Situación de los Derechos Humanos de las Mujeres en Guatemala. Guatemala, Nizkor, 2001. http://www.derechos.org/nizkor/guatemala/doc/sombra.html (30/05/2020)

ONU. Objetivos de Desarrollo sostenible No. 5. Nueva York, ONU, 2012. 20http://www.un.org/sustainabledevelopment/es/gender- equality/ (14/01/2020)

ONU. Por qué no tenemos paridad, Informe Atenea. Guatemala, Onumujeres, 2018. http://onu.org.gt/ wp-content/uploads/2018/08/InformeAtenea_2018.pdf (20/06/2020)

Organismo Judicial. Ley de Dignificación, Promoción Integral de la Mujer. Guatemala, José Pineda Ibarra, 1990.

PMA/CEPAL. "El Costo del Hambre": Análisis del Impacto Social y Económico de la desnutrición infantil en América Latina. Resultado del Estudio en Guatemala. Santiago de Chile, Cepal, 2000.

Rouanet, Rina y González, D. “Presupuesto y Proyectos con enfoque de género" Guatemala, Serviprensa, 2003.

SEGEPLAN. "Informe de Estado de Cumplimiento de la Declaración y Plataforma de Beijing". Guatemala, Segeplan, 2014.

SEGEPLAN. Políticas Pública de Desarrollo Integral de las Mujeres 2008-2023. Guatemala, Segeplan, 2008.

http://www.segeplan.gob.gt/downloads/clearinghouse/politicas_publicas/Derechos\%20Humanos/ Politica\%20Promoci\%C3\%B3n\%20\%20y\%20desarrollo\%20Mujeres\%202008-2023.pdf(8/06/2020)

Torres Ruiz, Gladys. "Feminismo y Derechos humanos en la ciudadanía de las mujeres" . México, ClMAC, 2009. https://cimacnoticias.com.mx/noticia/feminismo-y-dh-vitales-en-ciudadania-plena-de-las-mujeres/ (4/6/2020)

Trimiño Vásquez, Celina. Género y Educación, Revista Historia de la Educación en Latinoamérica - Vol. 17 No. 24, 2015 55-71, https://doi.org/10.19053/01227238.3300

Vásquez, Sofía, Galicia, Patricia y Monzón, Silvia; “Entre la Realidad y El Desafío: Mujeres y la Participación Política en Guatemala" (Guatemala, NDI/Onu Mujeres, 2013). 